\title{
Nonlinear Spinors as the Candidate of Dark Matter
}

\section{Yingqiu Gu}

School of Mathematical Science, Fudan University, Shanghai, China

Email: yqgu@fudan.edu.cn

How to cite this paper: Gu, Y.Q. (2017) Nonlinear Spinors as the Candidate of Dark Matter. Open Access Library Journal, 4: e3954.

https://doi.org/10.4236/oalib.1103954

Received: September 20, 2017

Accepted: October 23, 2017

Published: October 26, 2017

Copyright (๑) 2017 by author and Open Access Library Inc.

This work is licensed under the Creative Commons Attribution International License (CC BY 4.0).

http://creativecommons.org/licenses/by/4.0/

\begin{abstract}
In this paper, we discuss the equation of state for nonlinear spinor gases in the context of cosmology. The mean energy momentum tensor is similar to that of the prefect fluid, but an additional function of state $W$ is introduced to describe the nonlinear potential. The equation of state $w(a) \lesssim-1$ in the early universe is calculated, which provides a natural explanation for the negative pressure of dark matter and dark energy. $W$ may be also the main origin of the cosmological constant $\Lambda$. So the nonlinear spinor gases may be a candidate for dark matter and dark energy.
\end{abstract}

\section{Subject Areas}

Mathematical Analysis, Particle Physics

\section{Keywords}

Equation of State, Negative Pressure, Nonlinear Spinor, Dark Matter, Dark Energy

\section{Introduction}

Since the discovery that the expansion of the universe is accelerating [1]-[7], the standard model of cosmology has shifted from a matter dominated and decelerating expansion picture to search for the dark matter and dark energy with repulsive gravity or modification of the general relativity. The present methods to analyze the observational data include direct measures of cosmic scales through Type Ia supernova luminosity distances, the angular distance scales of baryon acoustic oscillation and cosmic microwave background density perturbations, as well as indirect probes such as the effect of cosmic expansion on the growth of matter density fluctuations. The basic building blocks of the 
universe are believed to consist of $4 \%$ baryons, $20 \%$ dark matter, and $76 \%$ dark energy. The cosmic accelerating expansion results in profound mysteries in all of science, with deep connections to both astrophysics and particle physics.

The simplest choice for such dark energy with negative pressure is the cosmological constant $\Lambda$, which consists with Einstein's equations. This term acts like a fluid with equation of state (EOS) $p_{\Lambda}=-\rho_{\Lambda}$. Although the $\Lambda$ CDM model is basically consistent with all observational data, the evaluated $\Lambda$ seems not coincident with the local Newtonian approximation of the galactic gravity, and a lot of theoretical efforts have been paid to explain its origin and apparent value [8] [9] [10] [11].

The widely studied dynamical models to describe dark energy are mainly the scalar fields, such as quintessence, K-essence, tachyon, phantom and dilaton, which are uniformly distributed in the universe [11]-[20]. These models have different theoretical origins and consequences, and some cases can give explanation for the observational data and the origin of $\Lambda$ [12] [19] [21].

Noting the facts that all fermions are described by spinors and the uniform scalar field can hardly explain the galactic structure [22], so the dark spinors may be partially responsible for dark matter. In this paper, we give a detailed discussion for the EOS of nonlinear spinor gas in the context of cosmology. Here the spinors are quantized and identified by nonlinear potentials, and their state functions are derived from normal Dirac equations. This work is a further research of the preceding papers [23] [24] [25]. Some similar works were once done in [26]-[31], where the authors also considered the nonlinear spinor field as candidate of dark energy, and only one spinor field was taken into account.

The nonlinear spinors provide us some interesting consequences and new insights: The negative pressure exists and we have the equation of state (EOS) $w(z) \sim-1$ in the early universe. The state functions are only the functions of scale factor but independent of its derivatives. The calculations show that the nonlinear spinors can give more natural explanation to the cosmic accelerating expansion and other observational data.

\section{Equation of State of Nonlinear Spinor Gas}

Define $4 \times 4$ Hermitian matrices as follows

$$
\alpha^{\mu}=\left\{\left(\begin{array}{cc}
I & 0 \\
0 & I
\end{array}\right),\left(\begin{array}{cc}
0 & \vec{\sigma} \\
\vec{\sigma} & 0
\end{array}\right)\right\}, \gamma=\left(\begin{array}{cc}
I & 0 \\
0 & -I
\end{array}\right)
$$

We consider the following Lagrangian for the same kind spinors in flat spacetime [23] [24]

$$
\mathcal{L}_{m}=\sum_{n}\left(\phi_{n}^{+}\left(\alpha^{\mu} i \partial_{\mu}-\mu \gamma\right) \phi_{n}+V_{n}\left(\breve{\gamma}_{n}\right)\right), \quad \breve{\gamma}_{n}=\phi_{n}^{+} \gamma \phi_{n}
$$

where $\phi_{n}$ stands for the $n$-th dark spinor, $\mu>0$ is constant with mass dimension, which takes one value for the same kind particles. $V_{n}\left(\breve{\gamma}_{n}\right)$ is the nonlinear potential term. 
The simplest case of dark spinor has the self potential $V_{n}=\frac{1}{2} \omega \breve{\gamma}_{n}^{2}$, in which $\omega>0$ is a constant. The dynamical equation for $n$-th spinor is given by

$$
\hbar i \partial_{t} \phi_{n}=\hat{H} \phi_{n}, \quad \hat{H}=c\left[\vec{\alpha} \cdot \hat{p}+\left(\mu c-\omega \breve{\gamma}_{n}\right) \gamma\right]
$$

In [24] [25] we computed the static mass $m_{n}$ and $w_{n}$ of a nonlinear spinor as the functions of spectrum parameter a (which cannot be confused with the following scale factor $a$ ),

$$
m_{n}=\frac{a^{2}-1}{a^{2}+1} \mu, \quad w_{n} \equiv \frac{1}{2} \omega \int_{0}^{\infty} \bar{\gamma}_{n}^{2} \cdot 4 \pi r^{2} \mathrm{~d} r=\frac{a \mu}{a^{2}+1} \frac{\int_{0}^{\infty}\left(u^{2}-v^{2}\right)^{2} \rho^{2} \mathrm{~d} \rho}{\int_{0}^{\infty}\left(u^{2}+v^{2}\right) \rho^{2} \mathrm{~d} \rho}
$$

For the ground state, by Figure 1 in [25] we have

$$
1<a<2, \quad E=\left(m_{n}+w_{n}\right) c^{2} \sim \mu c^{2}, \quad m_{n} \sim w_{n}
$$

For dark spinor gas (2.2), in microscopic view, the classical approximation of energy momentum tensor is given by [32]

$$
T^{\mu v}=\sum_{n}\left(m_{n} u_{n}^{\mu} u_{n}^{v}+w_{n} g^{\mu v}\right) \delta^{3}\left(\vec{x}-\vec{X}_{n}\right) \sqrt{1-v_{n}^{2}}
$$

where $u_{n}^{\mu}$ is the 4-vector velocity of $\phi_{n}, \vec{v}_{n}$ the usual 3-d speed, $\vec{X}_{n}(t)$ the central coordinate. The relativistic factor $\sqrt{1-v^{2}}$ is derived from the integral of Nöther charges [33]. The general form should be $\sqrt{1-g^{00} g_{k l} v^{k} v^{l}}$ in natural coordinate system [34]. In the case of linear spinor, we have the ideal gas model with $w_{n}=0,(\forall n)$, and then we have the usual energy-momentum tensor for perfect fluid. In case of $w_{n}>0$, the complete energy-momentum tensor should be

$$
T^{\mu v}=(\rho+P) U^{\mu} U^{v}+(W-P) g^{\mu v}
$$

where the additional term $W$ is a new function of state reflects the nonlinear potential of particles

$$
W=\frac{1}{V} \int_{V} \sum_{n} w_{n} \delta^{3}\left(\vec{x}-\vec{X}_{n}\right) \sqrt{1-v_{n}^{2}} \mathrm{~d} V=\frac{1}{V} \sum_{X_{n} \in V} w_{n} \sqrt{1-v_{n}^{2}}
$$

which acts like negative pressure. In the above equations we should distinguish the total energy density $T_{0}^{0}=\rho+W$ with the mass density $\rho$. They are different concepts for nonlinear spinor.

In a galaxy the usual pressure $P \sim 0$, by (2.5) we have $\rho \sim W$ and the total EOS in cosmology $w(z) \equiv P_{\text {tot }} / \rho_{\text {tot }} \sim-1$ in (2.7) [6] [7]. So the nonlinear spinor gas can give a natural explanation for the weird properties of dark matter and dark energy in category of normal field theory. Since $W$ take the place of $\Lambda$ in Einstein's field equation, it may be the main origin of the cosmological constant $\Lambda$. However the function of state $W$ is not a constant in cosmology, because the relativistic factor $\sqrt{1-v_{n}^{2}}$ is related to temperature $T$ [35], and $w_{n}$ is related to the scale factor. In what follows we give detailed analysis for these conclusions. 


\section{Relations and Equations in Cosmology}

In cosmology the scale factor $a(t)$ varies to very large range. In this case, the potential energy $w_{n}$ cannot be treated as constant. It should be the function of a. Now we derive $W(a)$ from the nonlinear Dirac equation coupling with Einstein equation.

In this paper, we adopt the conformal coordinate system for the universe, then the corresponding Friedmann-Robertson-Walker metric is given by

$$
g_{\mu \nu}=a^{2}(t) \operatorname{diag}\left[1,-1,-\mathcal{S}^{2}(r),-\mathcal{S}^{2}(r) \sin ^{2} \theta\right]
$$

where

$$
\mathcal{S}= \begin{cases}\sin r & \text { if } K=1, \\ r & \text { if } K=0, \\ \sinh r & \text { if } K=-1\end{cases}
$$

The dynamic Equation (2.3) for each spinor becomes

$$
\tilde{\alpha}^{\mu} i\left(\partial_{\mu}+\Upsilon_{\mu}\right) \phi_{n}=\left(\mu-V_{n}^{\prime}\right) \gamma \phi_{n}, \quad \Upsilon_{\mu}=\left(\frac{3 a^{\prime}}{2 a}, \frac{\mathcal{S}^{\prime}}{\mathcal{S}}, \frac{1}{2} \cot \theta, 0\right)
$$

where $\tilde{\alpha}^{\mu}$ is the coefficient matrix in curved space-time. It is easy to check the normalization condition holds for each spinor

$$
\int_{\Omega}\left|\phi_{n}\right|^{2} a^{3} \mathrm{~d} \Omega=1,(\forall n), \quad \mathrm{d} \Omega=\mathcal{S}^{2} \sin \theta \mathrm{d} r \mathrm{~d} \theta \mathrm{d} \varphi
$$

where $\mathrm{d} \Omega$ is the comoving volume element independent of $t$. Denote $\hat{p}^{v}=i\left(\partial^{v}+\Upsilon^{v}\right)$, the energy momentum tensor is given by [32]

$$
T^{\mu v}=\sum_{n}\left(\frac{1}{2} \Re\left\langle\phi_{n}^{+}\left(\tilde{\alpha}^{\mu} \hat{p}^{v}+\tilde{\alpha}^{v} \hat{p}^{\mu}\right) \phi_{n}\right\rangle+\left(V_{n}^{\prime} \breve{\gamma}_{n}-V_{n}\right) g^{\mu v}\right)
$$

where $\mathfrak{R}\langle\rangle$ means taking real part. The classical approximation of (3.5) is just (2.6).

In what follows we take $V_{n}=\frac{1}{2} \omega \breve{\gamma}_{n}^{2}$ as example to derive the equation of state $w(a)$ in usual sense. By normalizing condition (3.4) we learn that $\left|\phi_{n}\right|^{2} \rightarrow \delta\left(\vec{x}-\vec{X}_{n}\right)$, then in some sense we have $\vec{\gamma}_{n}^{2} \sim \delta^{2}\left(\vec{x}-\vec{X}_{n}\right)$ which depends on the scale of the space when space deforms seriously. To clarify this problem, making scaling transformation

$$
r=\frac{\bar{r}}{a_{0}}, \quad \phi_{n}=\left(\frac{a_{0}}{a(t)}\right)^{\frac{3}{2}} \psi_{n}
$$

where $a_{0}$ stands for the present scale factor.

Then we get

$$
V_{n}=\frac{\omega}{2}\left(\frac{a_{0}}{a}\right)^{6} \tilde{\gamma}_{n}^{2}
$$

in which $\tilde{\gamma}_{n} \equiv \psi_{n}^{+} \gamma \psi_{n}$. The normalization condition (3.4) becomes

$$
1=\int|\phi|^{2} a^{3} \mathrm{~d} \Omega=\int\left|\psi_{n}\right|^{2} a_{0}^{3} \mathrm{~d} \Omega
$$

so $\psi_{n}$ is normalized in the new coordinate system. By (2.8), (3.7) and (3.8) we 
can calculate the mean value of $W$ as

$$
W=\frac{1}{V} \sum_{X_{n} \in V} \int_{\Omega} V_{n} a^{3} \mathrm{~d} \Omega=\frac{1}{a^{3} \Omega} \sum_{X_{n} \in \Omega}\left(\frac{a_{0}}{a}\right)^{3} w_{n} \sqrt{1-v_{n}^{2}}=\frac{\rho \chi}{a^{3}}
$$

where $\rho=\frac{1}{\Omega} \sum_{X_{n} \in \Omega} m_{n}$ is the static comoving mass density of the spinors, $\chi=\chi(a)$ is a function defined by

$$
\chi=\left(\frac{a_{0}}{a}\right)^{3} \frac{\bar{w}}{\bar{m}}\left(1-\frac{3 \sigma k T}{2(\sigma \bar{m}+k T)}\right)
$$

$\bar{m}$ and $\bar{w}$ are mean mass and potential of all particles calculated in local Minkowski space-time, $0<\sigma<\frac{2}{3}$ is a parameter determined by energy distribution function. For Maxwell distribution [35], we have $\sigma=\frac{2}{5}$.

In [35], we derived the following relations in case $w_{n}=0$,

$$
\begin{gathered}
k T=\frac{\sigma \bar{m}}{a}\left(\sqrt{a^{2}+b^{2}}-a\right) \\
\rho=\frac{\rho}{a^{3}}\left(1+\frac{3 \sigma}{2 a}\left(\sqrt{a^{2}+b^{2}}-a\right)\right), \quad P=\frac{\rho \sigma b^{2}}{2 a^{4} \sqrt{a^{2}+b^{2}}}
\end{gathered}
$$

where $b$ is a constant determined by the initial temperature. Substituting (3.11) into (3.10), we get

$$
\chi=\left(\frac{a_{0}}{a}\right)^{3} \frac{\bar{w}}{\bar{m}}\left(1-\frac{3}{2} \sigma+\frac{3 \sigma a}{2 \sqrt{a^{2}+b^{2}}}\right)
$$

We have asymptotically $\chi \rightarrow \chi_{0}\left(a_{0} / a\right)^{3}$ if $a \ll a_{0}$, where $\chi_{0}>0$ is a constant.

By (2.7) we have $T_{v}^{\mu}=\operatorname{diag}(\rho+W, W-P, W-P, W-P)$. Substituting (3.11), (3.12) and (3.13) into $T_{v}^{\mu}$ we get the EOS of nonlinear spinor gas in cosmology

$$
w(a)=\frac{P-W}{\rho+W}=\frac{\sigma b^{2}-2 \chi a \sqrt{a^{2}+b^{2}}}{\sqrt{a^{2}+b^{2}}\left(2 \chi a+3 \sigma \sqrt{a^{2}+b^{2}}+2 a-3 \sigma a\right)}
$$

When $a \ll a_{0}$ or $\chi \rightarrow \infty$ we get $w \rightarrow-1$. This gives a natural explanation for the negative pressure in the early universe. (3.14) is an increasing function of a. This feature is consistent with the WMAP five-year data [36].

\section{Discussion and Conclusion}

According to the above calculation, we get some important consequences and insights.

1) By (2.7), for the nonlinear spinor gas, the nonlinear potential acts as both the negative pressure and positive energy in the energy momentum tensor $T^{\mu v}$. When the universe becomes very compact, the term $W$ forms the main part of $T^{\mu v}$, and then we have EOS $w(a) \sim-1$. The nonlinear potential may also give 
an explanation for the origin of cosmological constant $\Lambda$.

2) Although the dynamical equation of the spinors is more complex than that of the scalar fields, the state functions of the spinors are even relatively simpler due to the normalizing condition (3.8). These functions are only the functions of scale factor, but independent of its derivatives.

3) Different from the ideal gas and fluid model, mass-energy density of the spinors is not a monotone decreasing function of a. Spinors have strong responses to the heavily curved space-time. Some more deeper research should be done.

4) A spinor $\phi_{n}$ only has a local micro structure, so one spinor field can only describe one particle. Some works use one spinor field to describe global dark energy. This treatment may be inadequate, and more attention should be paid.

\section{Acknowledgements}

The author is grateful to his supervisors Prof. Ta-Tsien Li for encouragement.

\section{References}

[1] Riess, A.G., et al. (1998) Supernova Search Team. The Astronomical Journal, 116, 1009.

[2] Perlmutter, S., et al. (1999) Supernova Cosmology Project. The Astronomical Journal, 517, 565. https://doi.org/10.1086/307221

[3] Spergel, N., et al. (2003) WMAP. The Astrophysical Journal Supplement, 148, 175.

[4] Tegmark, M., et al. (2004) Cosmological parameters from SDSS and WMAP. Physical Review D, 69, Article ID: 103501. https://doi.org/10.1103/PhysRevD.69.103501

[5] Turner, M.S. Why Cosmologists Believe the Universe Is Accelerating. The Proceedings of Type Ia Supernovae: Theory and Cosmology, the University of Chicago, 29-31 October 1998, Cambridge, UK.

[6] Dunkley, J., et al. (2009) Five-Year Wilkinson Microwave Anisotropy Probe (WMAP) Observations: Likelihoods and Parameters from the WMAP Data. The Astrophysical Journal Supplement, 180, 306-329. https://doi.org/10.1088/0067-0049/180/2/306

[7] Bochner, B., Pappas, D. and Dong, M. (2015) Testing Lambda and the Limits of Cosmography with the Union2.1 Supernova Compilation. The Astronomical Journal, 814, 7. https://doi.org/10.1088/0004-637X/814/1/7

[8] Weinberg, S. (2001) The Cosmological Constant Problems. In: Cline, D.B., Ed., Sources and Detection of Dark Matter and Dark Energy in the Universe, Springer, Berlin, Heidelberg, 18-26.

[9] Sahni, V. (2002) The Cosmological Constant Problem and Quintessence. Classical and Quantum Gravity, 19, 3435-3448.

[10] Peebles, P.J.E. and Ratra, B. (2003) The Cosmological Constant and Dark Energy. Reviews of Modern Physics, 75, 559-606. https://doi.org/10.1103/RevModPhys.75.559

[11] Copeland, E.J., Sami, M. and Tsujikawa, S. (2006) Dynamics of Dark Energy. IJMPD, 15, 1753-1936.

[12] Padmanabhan, T. (2005) Dark Energy: The Cosmological Challenge of the Millen- 
nium. Current Science, 88, 1057.

[13] Uzan, J.P. (2007) The Acceleration of the Universe and the Physics behind It. General Relativity and Gravitation, 39, 307-342. https://doi.org/10.1007/s10714-006-0385-Z

[14] Lamon, R. and Woehr, A. (2010) Quintessence and (Anti-)Chaplygin Gas in Loop Quantum Cosmology. Physics Review D, 81, Article ID: 024026.

[15] Turner, M.S. and Huterer, D. (2007) Cosmic Acceleration, Dark Energy and Fundamental Physics. Journal of the Physical Society of Japan, 76, Article ID: 111015. https://doi.org/10.1143/JPSJ.76.111015

[16] Linder, E.V. (2008) Mapping the Cosmological Expansion. Reports on Progress in Physics, 71, Article ID: 056901. https://doi.org/10.1088/0034-4885/71/5/056901

[17] Usmani, A.A. (2008) The Dark Energy Equation of State. Monthly Notices of the Royal Astronomical Society: Letters, 386, L92-L95. https://doi.org/10.1111/j.1745-3933.2008.00468.x

[18] Frieman, J.A., Turner, M.S. and Huterer, D. (2008) Dark Energy and the Accelerating Universe. Annual Review of Astronomy and Astrophysics, 46, 385-432.

[19] Padmanabhan, T. (2008) Emergent Gravity and Dark Energy.

[20] Szydlowski, M. (2007) Cosmological Zoo-Accelerating Models with Dark Energy. JCAP, 0709, 007.

[21] Bonvin, C., Caprini, C. and Durrer, R. (2006) No-Go Theorem for n-Essence Dark Energy. Physical Review Letters, 97, Article ID: 081303.

[22] Gu, Y.Q. (2008) Stationary Spiral Structure and Collective Motion of the Stars in a Spiral Galaxy.

[23] Gu, Y.Q. (2007) A Cosmological Model with Dark Spinor Source. International Journal of Modern Physics A, 22, 4667-4678. https://doi.org/10.1142/S0217751X07037925

[24] Gu, Y.Q. (1998) Some Properties of the Spinor Soliton. Advances in Applied Clifford Algebras, 8, 17-29. https://doi.org/10.1007/BF03041923

[25] Gu, Y.Q. (2017) Some Characteristic Functions for the Eigen Solution of Nonlinear Spinor. Quantum Physics Letters, 6, 123-129. https://doi.org/10.18576/qpl/060208

[26] Saha, B. (2016) Spinor Field with Polynomial Nonlinearity in LRS Bianchi Type-I Spacetime. Canadian Journal of Physics, 94, 116. https://doi.org/10.1139/cjp-2015-0574

[27] Adanhounme, V., Adomou, A., Codo, F.P. and Hounkonnou, M.N. (2012) Nonlinear Spinor Field Equations in Gravitational Theory: Spherical Symmetric Soliton-Like Solutions.

[28] Ribas, M.O., et al. (2005) Fermions as Sources of Accelerated Regimes in Cosmology. Physical Review D, 72, Article ID: 123502. https://doi.org/10.1103/PhysRevD.72.123502

[29] Chua, Ch.K. and Wong, G.G. (2016) Study of Majorana Fermionic Dark Matter. Physics Review D, 94, Article ID: 035002.

[30] Vacaru, S.I. (2015) Spinor and Twistor Geometry in Einstein Gravity and Finsler Modifications. Advances in Applied Clifford Algebras, 25, 453-483. https://doi.org/10.1007/s00006-014-0513-x

[31] Obukhov, Y.N. and Hehl, F.W. (2012) Extended Einstein-Cartan Theory a la Diakonov: The Field Equations.

[32] Gu, Y.Q. (2006) The Vierbein Formalism and Energy-Momentum Tensor of Spi- 
nors.

[33] Gu, Y.Q. (2007) Local Lorentz Transformation and Mass-Energy Relation of Spinor.

[34] Gu, Y.Q. (2017) Natural Coordinate System in Curved Space-Time.

[35] Gu, Y.Q. (2017) Functions of State for Spinor Gas in General Relativity.

[36] Li, H., et al. (2008) On using the WMAP Distance Priors in Constraining the Time Evolving Equation of State of Dark Energy. APJ, 683, L1-L4.

Open Access Library

Submit or recommend next manuscript to OALib Journal and we will provide best service for you:

- Publication frequency: Monthly

- 9 subject areas of science, technology and medicine

- Fair and rigorous peer-review system

- Fast publication process

- Article promotion in various social networking sites (LinkedIn, Facebook, Twitter, etc.)

- Maximum dissemination of your research work

Submit Your Paper Online: Click Here to Submit

Or Contact service@oalib.com 